\title{
Optimization of Manufacturing of Operational Amplifier Manufactured by Using Field-effect Heterotransistor to Decrease Their Dimensions
}

\author{
E. L. Pankratov ${ }^{1,2}$ \\ ${ }^{1}$ Nizhny Novgorod State University, Russia \\ ${ }^{2}$ Nizhny Novgorod State Technical University, Russia
}

Copyright $@ 2019$ by authors, all rights reserved. Authors agree that this article remains permanently open access under the terms of the Creative Commons Attribution License 4.0 International License

\begin{abstract}
In this paper we introduce an approach to decrease dimensions of operational amplifier based on field-effect heterotransistors. Dimensions of the elements will be decreased due to manufacture heterostructure with specific structure, doping of required areas of the heterostruc-ture by diffusion or ion implantation and optimization of annealing of dopant and/or radiation defects.
\end{abstract}

Keywords Operational Amplifier, Increasing Integration Rate of Field-effect Heterotransistors, Optimization of Manufacturing

\section{Introduction}

In the present time density of elements of integrated circuits and their performance intensively increasing. Simultaneously with increasing of the density of these elements of integrated circuits their dimensions decreases. One way to decrease dimensions of these elements of these integrated circuit is manufacturing of these elements in thin-film heterostructures [1-4]. An alternative approach to decrease dimensions of the elements of integrated circuits is using laser and microwave types annealing [5-7]. Using these types of annealing leads to generation inhomogeneous distribution of temperature. Due to Arrhenius law the inhomogeneity of the diffusion coefficient and other parameters of process. The inhomogeneity gives us possibility to decrease dimensions of elements of integrated circuits. Changing of properties of electronic materials could be obtain by using radiation processing of these materials $[8,9]$.

In this paper we consider an operational amplifier based on field-effect heterotransistors described in Ref. [10] (see Fig.1). We assume, that the considered element has been manufactured in heterostructure from Fig. 1. The heterostructure consist of a substrate and an epitaxial layer. The epitaxial layer includes into itself several sections manufactured by using another materials. The sections have been doped by diffusion or ion implantation to generation into these sections required type of conductivity ( $n$ or $p$ ). In this paper we analyzed redistribution of dopant during annealing of dopant and/ or radiation defects to formulate conditions for decreasing of dimensions of the considered amplifier.

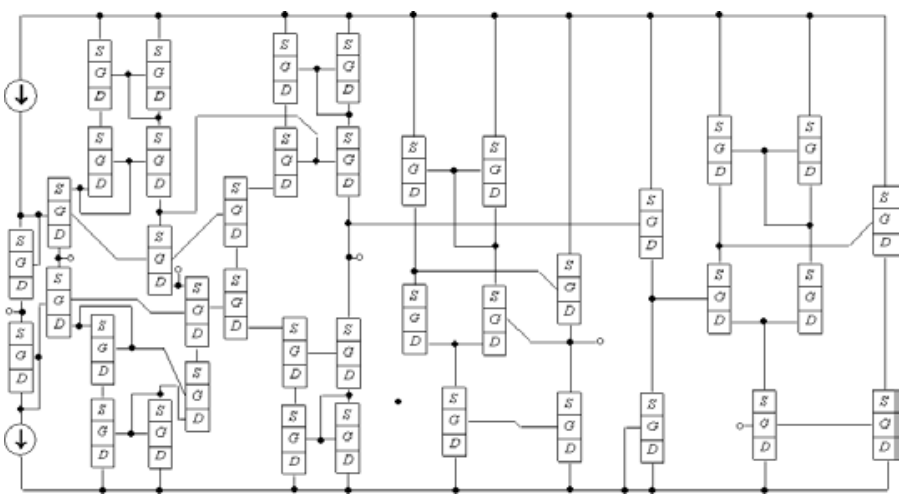

Figure 1a. Structure of the considered amplifier. View from top [10] 


\begin{tabular}{|l|l|l|l|l|l|l|l|}
\hline Source & Gate & Drain & & Source & Gate & Drain \\
\hline \multicolumn{1}{|l}{} \\
\hline
\end{tabular}

Figure 1b. Heterostructure with two layers and sections in the epitaxial layer

\section{Method of solution}

We determine spatio-temporal distribution of concentration of dopant by solving the following boundary problem

$$
\begin{aligned}
& \frac{\partial C(x, y, z, t)}{\partial t}= \\
& \quad=\frac{\partial}{\partial x}\left[D_{C} \frac{\partial C(x, y, z, t)}{\partial x}\right]+\frac{\partial}{\partial y}\left[D_{C} \frac{\partial C(x, y, z, t)}{\partial y}\right]+\frac{\partial}{\partial z}\left[D_{C} \frac{\partial C(x, y, z, t)}{\partial z}\right]
\end{aligned}
$$

with boundary and initial conditions

$$
\begin{array}{r}
\left.\frac{\partial C(x, y, z, t)}{\partial x}\right|_{x=0}=0,\left.\frac{\partial C(x, y, z, t)}{\partial x}\right|_{x=L_{x}}=0,\left.\frac{\partial C(x, y, z, t)}{\partial y}\right|_{y=0}=0, \\
\left.\frac{\partial C(x, y, z, t)}{\partial y}\right|_{x=L_{y}}=0,\left.\frac{\partial C(x, y, z, t)}{\partial z}\right|_{z=0}=0,\left.\frac{\partial C(x, y, z, t)}{\partial z}\right|_{x=L_{z}}=0, \mathrm{C}(\mathrm{x}, \mathrm{y}, \mathrm{z}, 0)=\mathrm{f} \quad(\mathrm{x}, \mathrm{y}, \mathrm{z}) .
\end{array}
$$

Here $C(x, y, z, t)$ is the spatio-temporal distribution of concentration of dopant; $T$ is the temperature of annealing; $D_{C}$ is the dopant diffusion coefficient. Value of dopant diffusion coefficient depends on properties of materials, speed of heating and cooling of heterostructure (with account Arrhenius law). Dependences of dopant diffusion coefficients could be approximated by the following function $[9,11,12]$

$$
D_{C}=D_{L}(x, y, z, T)\left[1+\xi \frac{C^{\gamma}(x, y, z, t)}{P^{\gamma}(x, y, z, T)}\right]\left[1+\varsigma_{1} \frac{V(x, y, z, t)}{V^{*}}+\varsigma_{2} \frac{V^{2}(x, y, z, t)}{\left(V^{*}\right)^{2}}\right],
$$

where $D_{L}(x, y, z, T)$ is the spatial (due to existing several layers wit different properties in heterostructure) and temperature (due to Arrhenius law) dependences of dopant diffusion coefficient; $P(x, y, z, T)$ is the limit of solubility of dopant; parameter $\gamma$ could be integer framework the following interval $\gamma \in[1,3]$ [9]; $V(x, y, z, t)$ is the spatio- temporal distribution of concentration of radiation vacancies; $V^{*}$ is the equilibrium distribution of concentration of vacancies. Concentrational dependence of dopant diffusion coefficient have been discussed in details in [9]. It should be noted, that using diffusion type of doping did not leads to generation radiation defects and $\zeta_{1}=\zeta_{2}=0$. We determine spatio-temporal distributions of concentrations of point defects have been determine by solving the following system of equations $[11,12]$

$$
\begin{gathered}
\frac{\partial I(x, y, z, t)}{\partial t}=\frac{\partial}{\partial x}\left[D_{I}(x, y, z, T) \frac{\partial I(x, y, z, t)}{\partial x}\right]+\frac{\partial}{\partial y}\left[D_{I}(x, y, z, T) \frac{\partial I(x, y, z, t)}{\partial y}\right]+ \\
+\frac{\partial}{\partial z}\left[D_{I}(x, y, z, T) \frac{\partial I(x, y, z, t)}{\partial z}\right]-k_{I, V}(x, y, z, T) I(x, y, z, t) V(x, y, z, t)- \\
-k_{I, I}(x, y, z, T) I^{2}(x, y, z, t)
\end{gathered}
$$




$$
\begin{array}{r}
\frac{\partial V(x, y, z, t)}{\partial t}=\frac{\partial}{\partial x}\left[D_{V}(x, y, z, T) \frac{\partial V(x, y, z, t)}{\partial x}\right]+\frac{\partial}{\partial y}\left[D_{V}(x, y, z, T) \frac{\partial V(x, y, z, t)}{\partial y}\right]+ \\
+\frac{\partial}{\partial z}\left[D_{V}(x, y, z, T) \frac{\partial V(x, y, z, t)}{\partial z}\right]-k_{I, V}(x, y, z, T) I(x, y, z, t) V(x, y, z, t)- \\
-k_{V, V}(x, y, z, T) V^{2}(x, y, z, t)
\end{array}
$$

with boundary and initial conditions

$$
\begin{aligned}
\left.\frac{\partial \rho(x, y, z, t)}{\partial x}\right|_{x=0}= & 0,\left.\frac{\partial \rho(x, y, z, t)}{\partial x}\right|_{x=L_{x}}=0,\left.\frac{\partial \rho(x, y, z, t)}{\partial y}\right|_{y=0}=0,\left.\frac{\partial \rho(x, y, z, t)}{\partial y}\right|_{y=L_{y}}=0, \\
& \left.\frac{\partial \rho(x, y, z, t)}{\partial z}\right|_{z=0}=0,\left.\frac{\partial \rho(x, y, z, t)}{\partial z}\right|_{z=L_{z}}=0, \rho(\mathrm{x}, \mathrm{y}, \mathrm{z}, 0)=\mathrm{f}_{\rho}(\mathrm{x}, \mathrm{y}, \mathrm{z}),
\end{aligned}
$$

Here $\rho=I, V ; I(x, y, z, t)$ is the spatio-temporal distribution of concentration of radiation interstitials; $D_{\rho}(x, y, z, T)$ is the diffusion coefficients of radiation interstitials and vacancies; terms $V^{2}(x, y, z, t)$ and $I^{2}(x, y, z, t)$ correspond to generation of divacancies and diinterstitials; $k_{I, V}(x, y, z, T)$ is the parameter of recombination of point radiation defects; $k_{\rho, \rho}(x, y, z, T)$ are the parameters of generation of simplest complexes of point radiation defects.

We determine spatio-temporal distributions of concentrations of divacancies $\Phi_{V}(x, y, z, t)$ and diinterstitials $\Phi_{I}(x, y, z, t)$ by solving the following system of equations [11,12]

$$
\begin{aligned}
& \frac{\partial \Phi_{I}(x, y, z, t)}{\partial t}=\frac{\partial}{\partial x}\left[D_{\Phi_{I}}(x, y, z, T) \frac{\partial \Phi_{I}(x, y, z, t)}{\partial x}\right]+\frac{\partial}{\partial y}\left[D_{\Phi_{I}}(x, y, z, T) \frac{\partial \Phi_{I}(x, y, z, t)}{\partial y}\right]+ \\
& +\frac{\partial}{\partial z}\left[D_{\Phi_{I}}(x, y, z, T) \frac{\partial \Phi_{I}(x, y, z, t)}{\partial z}\right]+k_{I, I}(x, y, z, T) I^{2}(x, y, z, t)-k_{I}(x, y, z, T) I(x, y, z, t) \\
& \frac{\partial \Phi_{V}(x, y, z, t)}{\partial t}=\frac{\partial}{\partial x}\left[D_{\Phi_{V}}(x, y, z, T) \frac{\partial \Phi_{V}(x, y, z, t)}{\partial x}\right]+\frac{\partial}{\partial y}\left[D_{\Phi_{V}}(x, y, z, T) \frac{\partial \Phi_{V}(x, y, z, t)}{\partial y}\right]+ \\
& +\frac{\partial}{\partial z}\left[D_{\Phi_{V}}(x, y, z, T) \frac{\partial \Phi_{V}(x, y, z, t)}{\partial z}\right]+k_{V, V}(x, y, z, T) V^{2}(x, y, z, t)-k_{V}(x, y, z, T) V(x, y, z, t)
\end{aligned}
$$

with boundary and initial conditions

$$
\begin{array}{r}
\left.\frac{\partial \Phi_{\rho}(x, y, z, t)}{\partial x}\right|_{x=0}=\left.0 \frac{\partial \Phi_{\rho}(x, y, z, t)}{\partial x}\right|_{x=L_{x}}, \quad=\left.0 \frac{\partial \Phi_{\rho}(x, y, z, t)}{\partial y}\right|_{y=0}=0, \\
\left.\frac{\partial \Phi_{\rho}(x, y, z, t)}{\partial y}\right|_{y=L_{y}}=0,\left.\frac{\partial \Phi_{\rho}(x, y, z, t)}{\partial z}\right|_{z=0}=0,\left.\frac{\partial \Phi_{\rho}(x, y, z, t)}{\partial z}\right|_{z=L_{z}}=0,
\end{array}
$$

$\Phi_{\mathrm{I}}(\mathrm{x}, \mathrm{y}, \mathrm{z}, 0)=\mathrm{f}_{\Phi \mathrm{I}}(\mathrm{x}, \mathrm{y}, \mathrm{z}), \Phi_{\mathrm{V}}(\mathrm{x}, \mathrm{y}, \mathrm{z}, 0)=\mathrm{f}_{\Phi V}(\mathrm{x}, \mathrm{y}, \mathrm{z})$. 
Here $D_{\varphi_{\rho}}(x, y, z, T)$ are the diffusion coefficients of complexes of radiation defects; $k_{\rho}(x, y, z, T)$ are the parameters of decay of complexes of radiation defects.

We determine spatio-temporal distributions of concentrations of dopant and radiation defects by using method of averaging of function corrections [13] with decreased quantity of iteration steps [14]. Framework the approach we used solutions of Eqs. (1), (4) and (6) in linear form and with averaged values of diffusion coefficients $D_{0 L}, D_{0 I}, D_{0 V}, D_{0 \Phi I}, D_{0 \phi V}$ as initial-order approximations of the required concentrations. The solutions could be written as

$$
\begin{array}{r}
C_{1}(x, y, z, t)=\frac{F_{0 C}}{L_{x} L_{y} L_{z}}+\frac{2}{L_{x} L_{y} L_{z}} \sum_{n=1}^{\infty} F_{n C} c_{n}(x) c_{n}(y) c_{n}(z) e_{n C}(t), \\
I_{1}(x, y, z, t)=\frac{F_{0 I}}{L_{x} L_{y} L_{z}}+\frac{2}{L_{x} L_{y} L_{z}} \sum_{n=1}^{\infty} F_{n I} c_{n}(x) c_{n}(y) c_{n}(z) e_{n I}(t) \\
V_{1}(x, y, z, t)=\frac{F_{0 C}}{L_{x} L_{y} L_{z}}+\frac{2}{L_{x} L_{y} L_{z}} \sum_{n=1}^{\infty} F_{n C} c_{n}(x) c_{n}(y) c_{n}(z) e_{n V}(t) \\
\Phi_{I 1}(x, y, z, t)=\frac{F_{0 \Phi_{I}}}{L_{x} L_{y} L_{z}}+\frac{2}{L_{x} L_{y} L_{z}} \sum_{n=1}^{\infty} F_{n \Phi_{I}} c_{n}(x) c_{n}(y) c_{n}(z) e_{n \Phi_{I}}(t) \\
\Phi_{V 1}(x, y, z, t)=\frac{F_{0 \Phi_{V}}}{L_{x} L_{y} L_{z}}+\frac{2}{L_{x} L_{y} L_{z}} \sum_{n=1}^{\infty} F_{n \Phi_{V}} c_{n}(x) c_{n}(y) c_{n}(z) e_{n \Phi_{V}}(t)
\end{array}
$$

where $e_{n \rho}(t)=\exp \left[-\pi^{2} n^{2} D_{0 \rho} t\left(\frac{1}{L_{x}^{2}}+\frac{1}{L_{y}^{2}}+\frac{1}{L_{z}^{2}}\right)\right], \quad F_{n \rho}=\int_{0}^{L_{x}} c_{n}(u) \int_{0}^{L_{y}} c_{n}(v) \int_{0}^{L_{z}} c_{n}(v) f_{\rho}(u, v, w) d w d v d u$, $c_{n}(\chi)=\cos \left(\pi n \chi / L_{\chi}\right)$.

With the second-order approximations and higher orders approximations of concentrations of dopant and radiation defects we determine framework for standard iterative procedure [13,14]. Framework of this procedure to calculate approximations with the $n$-th-order one shall replace the functions $C(x, y, z, t), I(x, y, z, t), V(x, y, z, t), \Phi_{I}(x, y, z, t), \Phi_{v}(x, y, z, t)$ in the right sides of the Eqs. (1), (4) and (6) on the following sums $\alpha_{n \rho}+\rho_{n-1}(x, y, z, t)$. As an example we present equations for the second-order approximations of the considered concentrations

$$
\begin{aligned}
& \frac{\partial C_{2}(x, y, z, t)}{\partial t}=\frac{\partial}{\partial x}\left(\left[1+\varsigma_{1} \frac{V(x, y, z, t)}{V^{*}}+\varsigma_{2} \frac{V^{2}(x, y, z, t)}{\left(V^{*}\right)^{2}}\right]\left\{1+\xi \frac{\left[\alpha_{2 C}+C_{1}(x, y, z, t)\right]^{\gamma}}{P^{\gamma}(x, y, z, T)}\right\} \times\right. \\
& \left.\times D_{L}(x, y, z, T) \frac{\partial C_{1}(x, y, z, t)}{\partial x}\right)+\frac{\partial}{\partial y}\left(D_{L}(x, y, z, T)\left[1+\varsigma_{1} \frac{V(x, y, z, t)}{V^{*}}+\varsigma_{2} \frac{V^{2}(x, y, z, t)}{\left(V^{*}\right)^{2}}\right] \times\right. \\
& \left.\times\left\{1+\xi \frac{\left[\alpha_{2 C}+C_{1}(x, y, z, t)\right]}{P^{\gamma}(x, y, z, T)}\right\} \frac{\partial C_{1}(x, y, z, t)}{\partial y}\right)+\frac{\partial}{\partial z}\left(D_{L}(x, y, z, T) \frac{\partial C_{1}(x, y, z, t)}{\partial z} \times\right. \\
& \left.\times\left[1+\varsigma_{1} \frac{V(x, y, z, t)}{V^{*}}+\varsigma_{2} \frac{V^{2}(x, y, z, t)}{\left(V^{*}\right)^{2}}\right]\left\{1+\xi \frac{\left.\xi \alpha_{2 C}+C_{1}(x, y, z, t)\right]^{\gamma}}{P^{\gamma}(x, y, z, T)}\right\}\right)
\end{aligned}
$$




$$
\begin{aligned}
& \frac{\partial I_{2}(x, y, z, t)}{\partial t}=\frac{\partial}{\partial x}\left[D_{I}(x, y, z, T) \frac{\partial I_{1}(x, y, z, t)}{\partial x}\right]+\frac{\partial}{\partial y}\left[D_{I}(x, y, z, T) \frac{\partial I_{1}(x, y, z, t)}{\partial y}\right]+ \\
& +\frac{\partial}{\partial z}\left[D_{I}(x, y, z, T) \frac{\partial I_{1}(x, y, z, t)}{\partial z}\right]-\left[\alpha_{2 I}+I_{1}(x, y, z, t)\right]\left[\alpha_{2 V}+V_{1}(x, y, z, t)\right] \times \\
& \times k_{I, V}(x, y, z, T)-k_{I, I}(x, y, z, T)\left[\alpha_{2 I}+I_{1}(x, y, z, t)\right]^{2} \\
& \frac{\partial V_{2}(x, y, z, t)}{\partial t}=\frac{\partial}{\partial x}\left[D_{V}(x, y, z, T) \frac{\partial V_{1}(x, y, z, t)}{\partial x}\right]+\frac{\partial}{\partial y}\left[D_{V}(x, y, z, T) \frac{\partial V_{1}(x, y, z, t)}{\partial y}\right]+ \\
& +\frac{\partial}{\partial z}\left[D_{V}(x, y, z, T) \frac{\partial V_{1}(x, y, z, t)}{\partial z}\right]-\left[\alpha_{2 I}+I_{1}(x, y, z, t)\right]\left[\alpha_{2 V}+V_{1}(x, y, z, t)\right] \times \\
& \times k_{I, V}(x, y, z, T)-k_{V, V}(x, y, z, T)\left[\alpha_{2 V}+V_{1}(x, y, z, t)\right]^{2} \\
& \frac{\partial \Phi_{I 2}(x, y, z, t)}{\partial t}=\frac{\partial}{\partial x}\left[D_{\Phi_{I}}(x, y, z, T) \frac{\partial \Phi_{I 1}(x, y, z, t)}{\partial x}\right]+\frac{\partial}{\partial y}\left[D_{\Phi_{I}}(x, y, z, T) \times\right. \\
& \left.\times \frac{\partial \Phi_{I 1}(x, y, z, t)}{\partial y}\right]+\frac{\partial}{\partial z}\left[D_{\Phi_{I}}(x, y, z, T) \frac{\partial \Phi_{I 1}(x, y, z, t)}{\partial z}\right]+k_{I, I}(x, y, z, T) \times \\
& \times I^{2}(x, y, z, t)-k_{I}(x, y, z, T) I(x, y, z, t) \\
& \frac{\partial \Phi_{V 2}(x, y, z, t)}{\partial t}=\frac{\partial}{\partial x}\left[D_{\Phi_{V}}(x, y, z, T) \frac{\partial \Phi_{V 1}(x, y, z, t)}{\partial x}\right]+\frac{\partial}{\partial y}\left[D_{\Phi_{V}}(x, y, z, T) \times\right. \\
& \left.\times \frac{\partial \Phi_{I 1}(x, y, z, t)}{\partial y}\right]+\frac{\partial}{\partial z}\left[D_{\Phi_{V}}(x, y, z, T) \frac{\partial \Phi_{V 1}(x, y, z, t)}{\partial z}\right]+k_{V, V}(x, y, z, T) \times \\
& \times V^{2}(x, y, z, t)-k_{V}(x, y, z, T) V(x, y, z, t)
\end{aligned}
$$

Integration of the left and right sides of Eqs.(8)-(10) gives us possibility to obtain relations for the second-order approximations of concentrations of dopant and radiation defects in the final forms

$$
\begin{aligned}
& C_{2}(x, y, z, t)=\frac{\partial}{\partial x}\left(\int_{0}^{t}\left[1+\varsigma_{1} \frac{V(x, y, z, \tau)}{V^{*}}+\varsigma_{2} \frac{V^{2}(x, y, z, \tau)}{\left(V^{*}\right)^{2}}\right]\left\{1+\xi \frac{\left[\alpha_{2 C}+C_{1}(x, y, z, \tau)\right]^{\gamma}}{P^{\gamma}(x, y, z, T)}\right\} \times\right. \\
& \left.\quad \times D_{L}(x, y, z, T) \frac{\partial C_{1}(x, y, z, \tau)}{\partial x}\right) d \tau+\frac{\partial}{\partial y}\left(\int_{0}^{t}\left[1+\varsigma_{1} \frac{V(x, y, z, \tau)}{V^{*}}+\varsigma_{2} \frac{V^{2}(x, y, z, \tau)}{\left(V^{*}\right)^{2}}\right] \times\right.
\end{aligned}
$$




$$
\begin{aligned}
& \left.\times D_{L}(x, y, z, T)\left\{1+\xi \frac{\left[\alpha_{2 C}+C_{1}(x, y, z, \tau)\right]^{\gamma}}{P^{\gamma}(x, y, z, T)}\right\} \frac{\partial C_{1}(x, y, z, \tau)}{\partial y} d \tau\right)+\frac{\partial}{\partial z}\left(\int_{0}^{t} D_{L}(x, y, z, T) \times\right. \\
& \left.\times\left[1+\varsigma_{1} \frac{V(x, y, z, \tau)}{V^{*}}+\varsigma_{2} \frac{V^{2}(x, y, z, \tau)}{\left(V^{*}\right)^{2}}\right]\left\{1+\xi \frac{\left[\alpha_{2 C}+C_{1}(x, y, z, \tau)\right]^{\gamma}}{P^{\gamma}(x, y, z, T)}\right\} \frac{\partial C_{1}(x, y, z, \tau)}{\partial z} d \tau\right)+ \\
& +f_{C}(x, y, z) \\
& I_{2}(x, y, z, t)=\frac{\partial}{\partial x}\left[\int_{0}^{t} D_{I}(x, y, z, T) \frac{\partial I_{1}(x, y, z, \tau)}{\partial x} d \tau\right]+\frac{\partial}{\partial y}\left[\int_{0}^{t} D_{I}(x, y, z, T) \times\right. \\
& \left.\times \frac{\partial I_{1}(x, y, z, \tau)}{\partial y} d \tau\right]+\frac{\partial}{\partial z}\left[\int_{0}^{t} D_{I}(x, y, z, T) \frac{\partial I_{1}(x, y, z, \tau)}{\partial z} d \tau\right]-\int_{0}^{t} k_{I, I}(x, y, z, T) \times \\
& \times\left[\alpha_{2 I}+I_{1}(x, y, z, \tau)\right]^{2} d \tau+f_{I}(x, y, z)-\int_{0}^{t} k_{I, V}(x, y, z, T)\left[\alpha_{2 I}+I_{1}(x, y, z, \tau)\right] \times \\
& \times\left[\alpha_{2 V}+V_{1}(x, y, z, \tau)\right] d \tau \\
& V_{2}(x, y, z, t)=\frac{\partial}{\partial x}\left[\int_{0}^{t} D_{V}(x, y, z, T) \frac{\partial V_{1}(x, y, z, \tau)}{\partial x} d \tau\right]+\frac{\partial}{\partial y}\left[\int_{0}^{t} D_{V}(x, y, z, T) \times\right. \\
& \left.\times \frac{\partial V_{1}(x, y, z, \tau)}{\partial y} d \tau\right]+\frac{\partial}{\partial z}\left[\int_{0}^{t} D_{V}(x, y, z, T) \frac{\partial V_{1}(x, y, z, \tau)}{\partial z} d \tau\right]-\int_{0}^{t} k_{V, V}(x, y, z, T) \times \\
& \times\left[\alpha_{2 I}+V_{1}(x, y, z, \tau)\right]^{2} d \tau+f_{V}(x, y, z)-\int_{0}^{t} k_{I, V}(x, y, z, T)\left[\alpha_{2 I}+I_{1}(x, y, z, \tau)\right] \times \\
& \times\left[\alpha_{2 V}+V_{1}(x, y, z, \tau)\right] d \tau \\
& \Phi_{I 2}(x, y, z, t)=\frac{\partial}{\partial x}\left[\int_{0}^{t} D_{\Phi_{I}}(x, y, z, T) \frac{\partial \Phi_{I 1}(x, y, z, \tau)}{\partial x} d \tau\right]+\frac{\partial}{\partial y}\left[\int_{0}^{t} D_{\Phi_{I}}(x, y, z, T) \times\right. \\
& \left.\times \frac{\partial \Phi_{I 1}(x, y, z, \tau)}{\partial y} d \tau\right]+\frac{\partial}{\partial z}\left[\int_{0}^{t} D_{\Phi_{I}}(x, y, z, T) \frac{\partial \Phi_{I 1}(x, y, z, \tau)}{\partial z} d \tau\right]+\int_{0}^{t} k_{I, I}(x, y, z, T) \times \\
& \times I^{2}(x, y, z, \tau) d \tau-\int_{0}^{t} k_{I}(x, y, z, T) I(x, y, z, \tau) d \tau+f_{\Phi_{I}}(x, y, z) \\
& \Phi_{V 2}(x, y, z, t)=\frac{\partial}{\partial x}\left[\int_{0}^{t} D_{\Phi_{V}}(x, y, z, T) \frac{\partial \Phi_{V 1}(x, y, z, \tau)}{\partial x} d \tau\right]+\frac{\partial}{\partial y}\left[\int_{0}^{t} D_{\Phi_{V}}(x, y, z, T) \times\right.
\end{aligned}
$$




$$
\begin{aligned}
\left.\times \frac{\partial \Phi_{I 1}(x, y, z, \tau)}{\partial y} d \tau\right] & +\frac{\partial}{\partial z}\left[\int_{0}^{t} D_{\Phi_{V}}(x, y, z, T) \frac{\partial \Phi_{V 1}(x, y, z, \tau)}{\partial z} d \tau\right]+\int_{0}^{t} k_{V, V}(x, y, z, T) \times \\
& \times V^{2}(x, y, z, \tau) d \tau-\int_{0}^{t} k_{V}(x, y, z, T) V(x, y, z, \tau) d \tau+f_{\Phi_{V}}(x, y, z)
\end{aligned}
$$

We determine average values of the second-orders approximations of the considered concentrations by using the following standard relations $[13,14]$

$$
\alpha_{2 \rho}=\frac{1}{\Theta L_{x} L_{y} L_{z}} \int_{0}^{\Theta} \int_{0}^{L_{x}} \int_{0}^{L_{y} L_{z}} \int_{0}\left[\rho_{2}(x, y, z, t)-\rho_{1}(x, y, z, t)\right] d z d y d x d t .
$$

Substitution of relations (8a)-(10a) into relation (11) gives us possibility to obtain relations for the required average values $\alpha_{2 \rho}$

$$
\begin{aligned}
& \alpha_{2 C}=\frac{1}{L_{x} L_{y} L_{z}} \int_{0}^{L_{x}} \int_{0}^{L_{y} L_{z}} \int_{0} f_{C}(x, y, z) d z d y d x \\
& \alpha_{2 I}=\frac{1}{2 A_{I I 00}}\left\{\left(1+A_{I V 01}+A_{I I 10}+\alpha_{2 V} A_{I V 00}\right)^{2}-4 A_{I I 00}\left[\alpha_{2 V} A_{I V 10}-A_{I I 20}+A_{I V 11}-\right.\right. \\
& \left.\left.-\frac{1}{L_{x} L_{y} L_{z}} \int_{0}^{L_{x}} \int_{0}^{L_{y}} \int_{0}^{L_{z}} f_{I}(x, y, z) d z d y d x\right]\right\}^{\frac{1}{2}}-\frac{1+A_{I V 01}+A_{I I 10}+\alpha_{2 V} A_{I V 00}}{2 A_{I I 00}} \\
& \alpha_{2 V}=\frac{1}{2 B_{4}} \sqrt{\frac{\left(B_{3}+A\right)^{2}}{4}-4 B_{4}\left(y+\frac{B_{3} y-B_{1}}{A}\right)}-\frac{B_{3}+A}{4 B_{4}} \\
& A_{a b i j}=\frac{1}{\Theta L_{x} L_{y} L_{z}} \int_{0}^{\Theta}(\Theta-t) \int_{0}^{L_{x}} \int_{0}^{L_{y} L_{z}} \int_{0} k_{a, b}(x, y, z, T) I_{1}^{i}(x, y, z, t) V_{1}^{j}(x, y, z, t) d z d y d x d t \\
& B_{4}=A_{I V 00}^{2} A_{I V 00}^{2}-2\left(A_{I V 00}^{2}-A_{I I 00} A_{V V 00}\right)^{2}, B_{3}=A_{I V 00} A_{I V 00}^{2}+A_{I V 01} A_{I V 00}^{3}+A_{I V 00} A_{I I 10} A_{I V 00}^{2}- \\
& -4\left(A_{I V 00}^{2}-A_{I I 00} A_{V V 00}\right)\left[2 A_{I V 01} A_{I V 00}+2 A_{I V 00}\left(1+A_{I V 01}+A_{I I 10}\right)-2 A_{I I 00}\left(A_{I V 10}+A_{V V 10}+1\right)\right]- \\
& -4 A_{I V 10} A_{I I 00} A_{I V 00}^{2}+2 A_{I V 00} A_{I V 01} A_{I V 00}^{2}, \quad B_{2}=A_{I V 00}^{2}\left\{\left(1+A_{I V 01}+A_{I I 10}\right)^{2}+A_{I V 00}^{2} A_{I V 01}^{2}+A_{I V 00} \times\right. \\
& \times 2 A_{I V 00}\left(A_{I V 00}+A_{I V 00} A_{I V 01}+A_{I V 00} A_{I I 10}-4 A_{I V 10} A_{I I 00}\right)-4 A_{I I 00}\left[A_{I V 11}-A_{I I 20}-\frac{1}{L_{x} L_{y} L_{z}} \times\right. \\
& \left.\left.\times \int_{0}^{L_{x}} \int_{0}^{L_{y}} \int_{0}^{L_{z}} f_{I}(x, y, z) d z d y d x\right]\right\}\left\{\left[2 A_{I V 01} A_{I V 00}+2 A_{I V 00}\left(1+A_{I V 01}+A_{I I 10}\right)-2 A_{I I 00}\left(A_{I V 10}+1+\right.\right.\right.
\end{aligned}
$$


$\left.\left.+A_{V V 10}\right)\right]^{2}+2\left[A_{I V 01}\left(1+A_{I V 01}+A_{I I 10}\right)+\frac{2}{L_{x} L_{y} L_{z}} \int_{0}^{L_{x}} \int_{0}^{L_{y}} \int_{0}^{L_{z}} f_{V}(x, y, z) d z d y d x-2 A_{I I 00}\left(A_{V V 20}-\right.\right.$ $\left.\left.-A_{I V 11}\right)+A_{I V 01}\left(1+A_{I V 01}+A_{I I 10}\right)\right]\left[2 A_{I V 00}\left(1+A_{I V 01}+A_{I I 10}\right)+2 A_{I V 01} A_{I V 00}-2 A_{I I 00}\left(A_{I V 10}+1+\right.\right.$ $\left.\left.\left.+A_{V V 10}\right)\right]\right\}$ $B_{1}=2 A_{I V 00} A_{I V 01}\left(1+A_{I V 01}+A_{I I 10}\right)^{2}-8\left[A_{I V 11}-\frac{1}{L_{x}} \int_{0}^{L_{x}} \int_{0}^{L_{y}} \int_{0}^{L_{z}} f_{I}(x, y, z) d z d y d x \times\right.$ $\left.\times \frac{1}{L_{y} L_{z}}-A_{I I 20}\right]+A_{I V 00} A_{I V 01} A_{I I 00}+A_{I V 01}^{2}\left(A_{I V 00}+A_{I V 00} A_{I V 01}+A_{I V 00} A_{I I 10}-4 A_{I V 10} A_{I I 00}\right)-$ $-2\left[\frac{2 A_{I I 00}}{L_{x} L_{y} L_{z}} \int_{0}^{L_{x}} \int_{0}^{L_{y}} \int_{0}^{L_{z}} f_{I}(x, y, z) d z d y d x+A_{I V 01}\left(1+A_{I V 01}+A_{I I 10}\right)-2 A_{I I 00}\left(A_{V V 20}-A_{I V 11}\right)+\right.$ $\left.+A_{I V 01}\left(1+A_{I V 01}+A_{I I 10}\right)\right]\left[2 A_{I V 00}\left(1+A_{I V 01}+A_{I I 10}\right)-2\left(A_{I V 10}+A_{V V 10}+1\right) A_{I I 00}+2 A_{I V 01} A_{I V 00}\right]$, $B_{0}=4 A_{I I 00} A_{I V 01}^{2}\left[A_{I I 20}+\frac{1}{L_{x} L_{y} L_{z}} \int_{0}^{L_{x}} \int_{0}^{L_{y}} \int_{0}^{L_{z}} f_{I}(x, y, z) d z d y d x-A_{I V 11}\right]+A_{I V 01}^{2}\left(A_{I V 01}+A_{I I 10}+\right.$ $+1)^{2}-\left[\frac{2 A_{I I 00}}{L_{x} L_{y} L_{z}} \int_{0}^{L_{x} L_{y}} \int_{0}^{L_{z}} \int_{0} f_{V}(x, y, z) d z d y d x+A_{I V 01}\left(1+A_{I V 01}+A_{I I 10}\right)-2 A_{I I 00}\left(A_{V V 20}-A_{I V 11}\right)+\right.$ $\left.+A_{I V 01}\left(1+A_{I V 01}+A_{I I 10}\right)\right]^{2}, y=\sqrt[3]{\sqrt{q^{2}+p^{3}}-q}-\sqrt[3]{\sqrt{q^{2}+p^{3}}+q}+\frac{B_{2}}{6}, q=\left(2 B_{1} B_{3}-8 B_{0}\right) \times$ $\times \frac{B_{2}}{48}+\frac{B_{2}^{3}}{216}+\frac{B_{0}\left(4 B_{2}-B_{3}^{2}\right)-B_{1}^{2}}{8}, p=\left[3\left(2 B_{1} B_{3}-8 B_{0}\right)-2 B_{2}^{2}\right] / 72, A=\sqrt{8 y+B_{3}^{2}-4 B_{2}}$, $\alpha_{2 \Phi_{I}}=A_{I I 20}-\frac{1}{\Theta L_{x} L_{y} L_{z}} \int_{0}^{\Theta}(\Theta-t) \int_{0}^{L_{x}} \int_{0}^{L_{y}} \int_{0}^{L_{z}} k_{I}(x, y, z, T) I(x, y, z, t) d z d y d x d t+$ $+\frac{1}{L_{x} L_{y} L_{z}} \int_{0}^{L_{x}} \int_{0}^{L_{y}} \int_{0}^{L_{z}} f_{\Phi I}(x, y, z) d z d y d x$

$$
\begin{aligned}
& \alpha_{2 \Phi_{V}}=A_{V 20}-\frac{1}{\Theta L_{x} L_{y} L_{z}} \int_{0}^{\Theta}(\Theta-t) \int_{0}^{L_{x}} \int_{0}^{L_{y}} \int_{0}^{L_{z}} k_{V}(x, y, z, T) V(x, y, z, t) d z d y d x d t+ \\
& +\frac{1}{L_{x} L_{y} L_{z}} \int_{0}^{L_{x}} \int_{0}^{L_{y}} \int_{0}^{L_{z}} f_{\Phi V}(x, y, z) d z d y d x
\end{aligned}
$$


The considered substitution gives us possibility to obtain equation for parameter $\alpha_{2 C}$. Solution of the equation depends on value of parameter $\gamma$. Analysis of spatio-temporal distributions of concentrations of dopant and radiation defects has been done by using their second-order approximations framework the method of averaged of function corrections with decreased quantity of iterative steps. The second-order approximation is usually good enough approximation to make qualitative analysis and obtain some quantitative results. Results of analytical calculation have been checked by comparison with results of numerical simulation.

\section{Discussion}

In this section we analyzed the spatio-temporal distribution of concentration of dopant in the considered heterostructure during annealing. Figs. 2 shows spatial distributions of concentrations of dopants infused (Fig. 2a) or implanted (Fig. $2 b$ ) in epitaxial layer. Value of annealing time is equal for all distributions framework every figure $2 a$ and $2 b$. Increasing of number of curves corresponds to increasing of difference between values of dopant diffusion coefficients in layers of heterostructure. The figures show that presents of interface between layers of heterostructure gives us possibility to increase absolute value of gradient of concentration of dopant in direction, which is perpendicular to the interface. We obtain increasing of absolute value of the gradient in neighborhood of the interface. Due to the increasing one can obtain decreasing dimensions of transistors, which have been used in the amplifier. At the same time with increasing of the gradient homogeneity of concentration of dopant in enriched area increases.

To choose annealing time it should be accounted with decreasing of absolute value of gradient of concentration of dopant in neighborhood of interface between substrate and epitaxial layer with increasing of annealing time. Decreasing of value of annealing time leads to decreasing of homogeneity of concentration of dopant in enriched area (see Fig. $3 a$ for diffusion doping of materials and Fig. $3 b$ for ion doping of materials). Let us determine compromise value of annealing time framework recently introduced criteria [15-20]. Framework this criteria we approximate real distributions of concentration of dopant by ideal rectangle distribution $\psi(x, y, z)$. Farther we determine compromise value of annealing time by minimization of the mean-squared error

$$
U=\frac{1}{L_{x} L_{y} L_{z}} \int_{0}^{L_{x} L_{y} L_{z}} \int_{0}^{0}[C(x, y, z, \Theta)-\psi(x, y, z)] d z d y d x .
$$

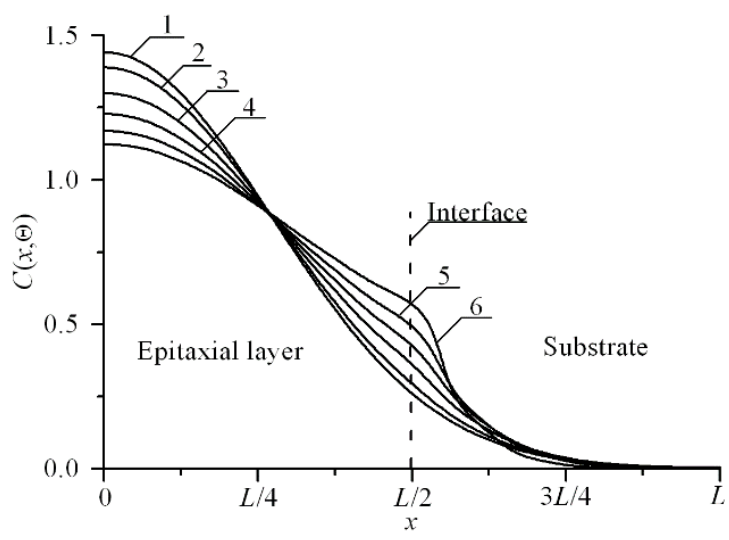

Figure2a. Distributions of concentration of infused dopant in heterostructure from Figs. 1 and 2 in direction, which is perpendicular to interface between epitaxial layer substrate. Increasing of number of curve corresponds to increasing of difference between values of dopant diffusion coefficient in layers of heterostructure under condition, when value of dopant diffusion coefficient in epitaxial layer is larger, than value of dopant diffusion coefficient in substrate

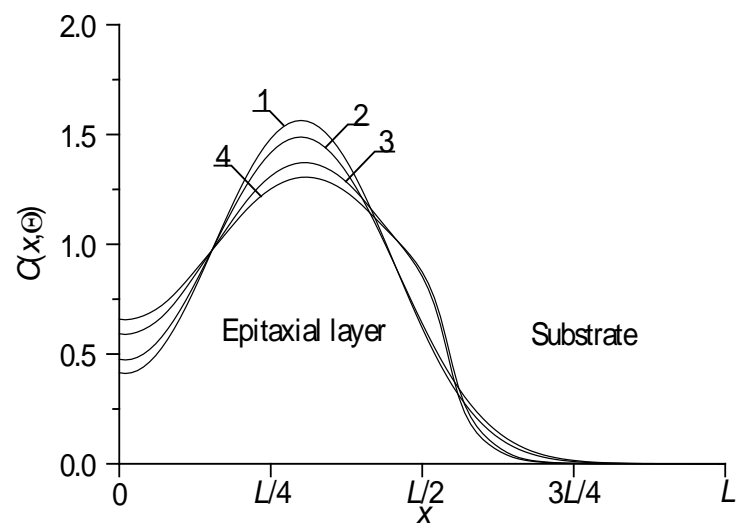

Figure2b. Distributions of concentration of implanted dopant in heterostructure from Figs. 1 and 2 in direction, which is perpendicular to interface between epitaxial layer substrate. Curves 1 and 3 corresponds to annealing time $\Theta=0.0048\left(L_{x}{ }^{2}+L_{y}{ }^{2}+L_{z}{ }^{2}\right) / D_{0}$. Curves 2 and 4 corresponds to annealing time $\Theta=0.0057\left(L_{x}{ }^{2}+L_{y}{ }^{2}+L_{z}{ }^{2}\right) / D_{0}$. Curves 1 and 2 corresponds to homogenous sample. Curves 3 and 4 corresponds to heterostructure under condition, when value of dopant diffusion coefficient in epitaxial layer is larger, than value of dopant diffusion coefficient in substrate

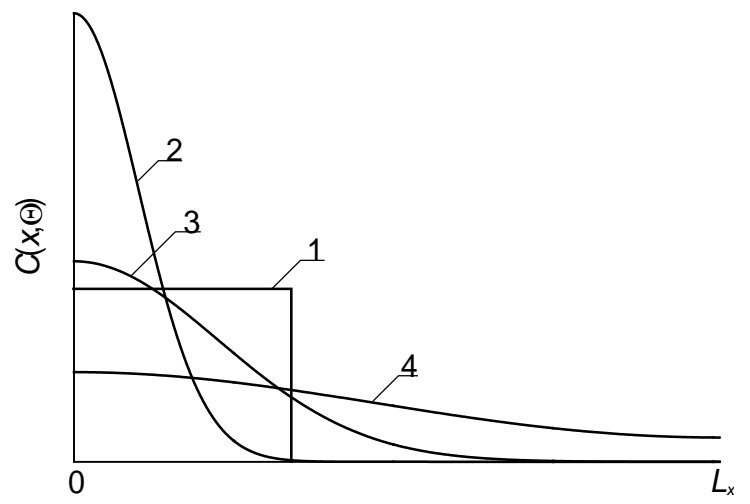

Figure 3a. Spatial distributions of dopant in heterostructure after dopant infusion. Curve 1 is idealized distribution of dopant. Curves 2-4 are real distributions of dopant for different values of annealing time. Increasing of number of curve corresponds to increasing of annealing time 


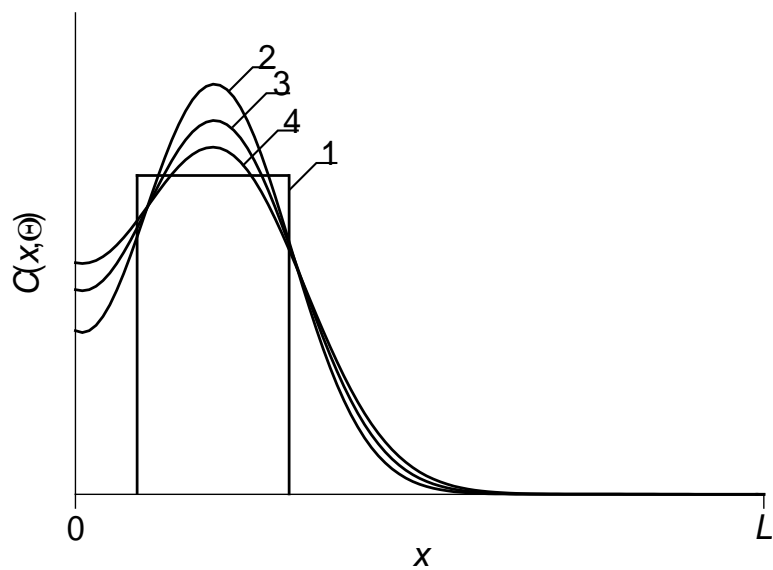

Figure3b. Spatial distributions of dopant in heterostructure after ion implantation. Curve 1 is idealized distribution of dopant. Curves 2-4 are real distributions of dopant for different values of annealing time. Increasing of number of curve corresponds to increasing of annealing time

Dependences of optimal annealing time are presented on Figs. 4 for diffusion and ion types of doping, respectively. It should be noted, that it is necessary to anneal radiation defects after ion implantation. One could find spreading of concentration of distribution of dopant during this annealing. In the ideal case distribution of dopant achieves appropriate interfaces between materials of heterostructure during annealing of radiation defects. If dopant did not achieves any interfaces during annealing of radiation defects, it is practicably to additionally anneal the dopant. In this situation optimal value of additional annealing time of implanted dopant is smaller, than annealing time of infused dopant. At the same time ion type of doping gives us possibility to decrease mismatch-induced stress in heterostructure [21].

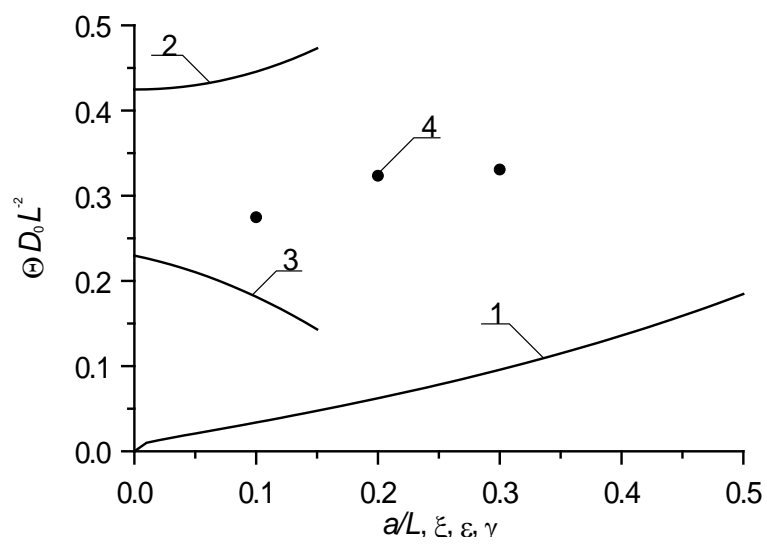

Figure4a. Dependences of dimensionless optimal annealing time for doping by diffusion, which have been obtained by minimization of mean-squared error, on several parameters. Curve 1 is the dependence of dimensionless optimal annealing time on the relation $a / L$ and $\xi=\gamma=0$ for equal to each other values of dopant diffusion coefficient in all parts of heterostructure. Curve 2 is the dependence of dimensionless optimal annealing time on value of parameter $\varepsilon$ for $a / L=1 / 2$ and $\xi=\gamma=0$. Curve 3 is the dependence of dimensionless optimal annealing time on value of parameter $\xi$ for $a / L=1 / 2$ and $\varepsilon=\gamma=0$. Curve 4 is the dependence of dimensionless optimal annealing time on value of parameter $\gamma$ for $a / L=1 / 2$ and $\varepsilon=\xi=0$

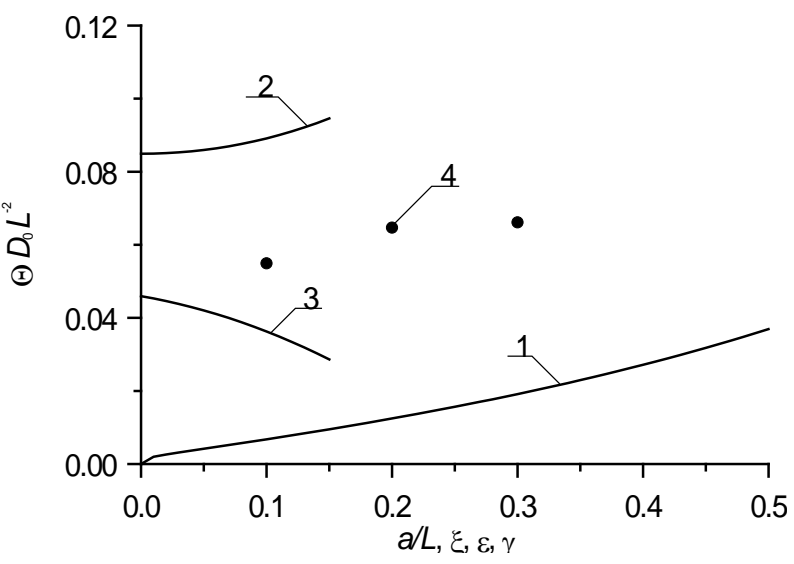

Figure4b. Dependences of dimensionless optimal annealing time for doping by ion implantation, which have been obtained by minimization of mean-squared error, on several parameters. Curve 1 is the dependence of dimensionless optimal annealing time on the relation $\mathrm{a} / \mathrm{L}$ and $\xi=\gamma=0$ for equal to each other values of dopant diffusion coefficient in all parts of heterostructure. Curve 2 is the dependence of dimensionless optimal annealing time on value of parameter $\varepsilon$ for $\mathrm{a} / \mathrm{L}=1 / 2$ and $\xi=\gamma=0$. Curve 3 is the dependence of dimensionless optimal annealing time on value of parameter $\xi$ for $\mathrm{a} / \mathrm{L}=1 / 2$ and $\varepsilon=\gamma=0$. Curve 4 is the dependence of dimensionless optimal annealing time on value of parameter $\gamma$ for $\mathrm{a} / \mathrm{L}=1 / 2$ and $\varepsilon=\xi=0$

\section{Conclusions}

In this paper we model redistribution of infused and implanted dopants during manufacture of operational amplifier based on field-effect heterotransistors. Several recommendations to optimize manufacturing process of the heterotransistors have been formulated. Analytical approach to model diffusion and ion types of doping with account changing of parameters in space and at the same time in time has been introduced. At the same time the approach gives us possibility to take into account nonlinearity of doping processes.

\section{REFERENCES}

[1] G. Volovich, Modern Electronics, Issue 2, 10 (2006).

[2] A. Kerentsev, and V. Lanin, Power Electronics, Issue 1, 34 (2008).

[3] A.O. Ageev, A.E. Belyaev, N.S. Boltovets, V.N. Ivanov, R.V. Konakova, Ya.Ya. Kudrik, P.M. Litvin, V.V. Milenin, and A.V. Sachenko, Semiconductors, 43, 897 (2009).

[4] N.I. Volokobinskaya, I.N. Komarov, T.V. Matioukhina, V.I. Rechetniko, A.A. Rush, I.V. Falina, and A.S. Yastre-bov, Semiconductors, 35, 1013 (2001).

[5] K.K. Ong, K.L. Pey, P.S. Lee, A.T.S. Wee, X.C. Wang, and Y.F. Chong, Appl. Phys. Lett., 89, 172111 (2006).

[6] H.T. Wang, L.S. Tan, and E.F. Chor, J. Appl. Phys., 98, 
094901 (2006).

[7] Yu.V. Bykov, A.G. Yeremeev, N.A. Zharova, I.V. Plotnikov, K.I. Rybakov, M.N. Drozdov, Yu.N. Drozdov, and V.D. Skupov, Radiophysics and Quantum Electronics, 43, 836 (2003).

[8] V.V. Kozlivsky, Modification of semiconductors by proton beams, Nauka, Sant-Peterburg (2003).

[9] V.L. Vinetskiy, and G.A. Kholodar', Radiative physics of semiconductors, "Naukova Dumka", Kiev (1979).

[10] K.L. Pushkar, K. Gupta, and P. Vivek, Circuits and Systems, 8, 275 (2017).

[11] Z.Yu. Gotra, Technology of microelectronic devices, Radio and communication, Moscow (1991).

[12] P.M. Fahey, P.B. Griffin, and J.D. Plummer, Rev. Mod. Phys., 61, 289 (1989).
[13] Yu.D. Sokolov, Applied Mechanics, 1, 23 (1955).

[14] E.L. Pankratov, Eur. Phys. J. B, 57, 251 (2006).

[15] E.L. Pankratov, Russian Microelectronics, 36, 33 (2007).

[16] E.L. Pankratov, Int. J. Nanoscience, 7, 187 (2008).

[17] E.L. Pankratov. J. Comp. Theor. Nanoscience, 7, 289 (2010).

[18] E.L. Pankratov, and E.A. Bulaeva, J. Comp. Theor. Nanoscience, 10, 888 (2013).

[19] E.L. Pankratov, and E.A. Bulaeva, Int. J. Micro-Nano Scale Transp., 4, 17 (2014).

[20] E.L. Pankratov, and E.A. Bulaeva, Int. J. Nanoscience, 11, 1250028 (2012).

[21] E.L. Pankratov, and E.A. Bulaeva, J. Comp. Theor. Nanoscience, 11, 91 (2014). 